Como citar este artigo: NASCIMENTO, IRAMAR B.; FLEIC, RAQUEL.

Identificação dos fatores que dificultam a interrupção do coronavírus (COVID-19) no Brasil. Revista Saúde (Sta. Maria). 2020; 46 (2).

Autor correspondente:

Nome: Raquel Fleig

E-mail: raquel.fleig@udesc.br

Telefone: (47) 9900-7466

Formação Profissional:

Doutoranda no Programa de

Pos-Craduacao em Planejamento

Territorial e Desenvolvimento

Socioambiental - FAED/UDESC -

São Bento do Sul (SC), Brasil.

Filiação Institucional: Universidade do Estado de Santa Catarina

Endereço para correspondencia:

Rua: Pascoal Simone

Bairro: Coqueiros

Cidade: Florianópolis

Estado: Santa Catarina

CEP: 88080-350

Data de Submissão:

04/05/2020

Data de aceite:

30/10/2020

Conflito de Interesse: Não há conflito de interesse

(cc) $\mathrm{BY}-\mathrm{NC}-\mathrm{ND}$

\section{Identificação dos fatores que dificultam a interrupção do coronavírus (COVID-19) no Brasil}

\section{Identification of factors that difficult the coronavirus (COVID-19) interruption in Brazil}

\author{
Iramar Baptistella do Nascimento, Raquel Fleig
}

\section{RESUMO}

A COVID-19 se propaga de forma exponencial, cujas taxas de transmissibilidade apresentam uma ascendência vertical que preocupa o mundo da saúde. 0 objetivo desse estudo foi o de identificar os fatores que dificultam a interrupção do coronavírus (COVID-19) no Brasil. Revisão integrativa da literatura nas bases de dados PubMed/MEDLINE, Web of Science, Scopus, LILACS e Cochrane. Esta revisão baseou-se nos critérios do cheklistPRISMA. Vinte nove (29)estudos estavam dentro dos critérios estabelecidos. Os temas avaliados foram: taxas de letalidade e parâmetros de transmissibilidade, resposta da vigilância nacional efetiva da doença, propagação dos patógenos humanos do SarsCoV-2 no cenário mundial e no Brasil e os recursos para interromper a transmissibilidade da patologia. A COVID-19 apresenta uma rápida ascensão e a missão que se atribui aos nossos governantes parece ser a reavaliação para converter a ascensão vertical. Profissionais capacitados à exposição de agentes patogênicos, estratégias de aconselhamento psicológico aos familiares e equipes multidisciplinares com maior preparo dificultam a desaceleração do coronavírus no Brasil. É necessário preconizar 0 destino das verbas para a capacitação de profissionais e compra de novos equipamentos que permitam o acompanhamento dos resultados no período do dia e/ou no tempo real, uma vez que no atual cenário as taxas de letalidade são ascendentes. Portanto, é de fundamental importância a ação de resguardo à segurança dos profissionais e agilizar a identificação dos pacientes sintomáticos à COVID-19 e dos indivíduos envolvidos, posto que a sua disseminação e/ou celeridade epidemiológica deve ser contida com a mesma força que ela é produzida.

PALAVRAS-CHAVE: Coronavírus; Pandemia; Prevenção de doenças.

\section{ABSTRACT}

COVID-19 spreads exponentially, whose transmissibility rates have a vertical ascendancy that worries the health world. The aim of this study was to identify the factors that hinder the interruption of the coronavirus (COVID-19) in Brazil. Integrative literature review in the PubMed / MEDLINE, Web of Science, Scopus, LILACS and Cochrane databases. This review was based on the criteria of the PRISMA checklist. Twenty nine (29) studies were within the established criteria. The topics evaluated were: lethality rates and transmissibility parameters, response to effective national surveillance of the disease, the spread of human Sars-CoV-2 pathogens on the world stage and in Brazil, and the resources to stop the transmissibility of the pathology. COVID-19 shows a rapid rise and the mission that is attributed to our leaders seems to be the reevaluation to convert the vertical rise. Professionals trained in the exposure of pathogens, psychological counseling strategies to family members and multidisciplinary teams with greater preparation make it difficult to decelerate the coronavirus in Brazil. It is necessary to recommend the destination of the funds for the training of professionals and the purchase of new equipment that allows the monitoring of the results in the period of the day and / or in real time, since in the current scenario, the lethality rates are rising. Therefore, it is of fundamental importance to safeguard the safety of professionals and to speed up the identification of patients symptomatic to COVID-19 and the individuals involved, since its dissemination and / or epidemiological speed must be contained with the same strength as it is produced. 


\section{INTRODUÇÃO}

O mundo se defronta com uma emergência de saúde pública internacional. A Sars-CoV-2 conhecida como doença do coronavírus 2019 (COVID-19) foi relatada em dezembro de 2019 em Wuhan, China¹. A doença apresenta relações genéticas com a Sars-Cov, que embora sua epidemia em 25 países tenha sido contida com sucesso em 2002-2003 pelas estratégias de isolamento e intervenções, não impediu o seu amplo reaparecimento e disseminação de forma pandêmica em um cenário global ${ }^{1,2}$.

As taxas de transmissão são um obstáculo a ser vencido, uma vez que prevalece um despreparo de diferentes costumes e culturas de populações humanas para se adequar a uma nova rotina que envolve um diferente perfil e um conjunto de métodos e estratégias capazes de proteger o organismo contra a proliferação da COVID-19². Outra questão está relacionada ao impacto para os países de média e baixa renda per capita, uma vez que os reflexos das informações que se decorrem dos meios de comunicação, até mesmo nos países de primeiro mundo, apontam a escassez de equipamentos para 0 atendimento de pacientes mais graves, tanto o déficit de acessórios de proteção individual para equipe de saúde quanto a falta de ventiladores respiratórios hospitalares, frente ao possível aumento da pandemia ${ }^{3,4,5}$.

Tais circunstâncias vêm causando preocupações na comunidade médica, bem como a forma como o vírus está se espalhando e o quadro mutável epidemiológico a cada dia ${ }^{2,6}$. Relatos demarcam a rapidez de como a doença se propaga, apontando em alguns casos de pessoas saudáveis, uma dor de garganta, e já no dia seguinte, uma febre de $39,1^{\circ} \mathrm{C}\left(102,4^{\circ} \mathrm{F}\right)$, somada a uma tosse seca ${ }^{6}$.

Pacientes assintomáticos se privilegiaram de uma breve recuperação. No entanto, o que despertou sentimento de apreensão e temeridade ao mundo da saúde foi principalmente quanto à prevalência da alta carga viral da COVID-19 nestes pacientes reestabelecidos ${ }^{6,7}$.

As preocupações dos cientistas e profissionais da saúde são consistentes e justificáveis frente às dificuldades de combater a COVID-19². Já é notória a necessidade de reavaliação sobre a dinâmica de transmissão do surto contemporâneo, levando em consideração os diferentes aspectos e incertezas, até mesmo nos pacientes convalescentes, e a possível viabilidade da COVID-19 nestes pacientes, uma vez que se torna necessária a comprovação em dados quantitativos de ensaio reverse-transcriptase-polymerase-chain-reaction (qRT-PCR) ${ }^{7,8}$. Desta forma, o objetivo deste estudo é identificar os fatores que dificultam a interrupção do coronavírus (COVID-19) no Brasil.

\section{MÉTODOS}

Realizou-se uma revisão integrativa da literatura entre os dias 3 de janeiro de 2020 e 3 abril de 2020. Organizou-se um protocolo envolvendo um relatório de avaliação com diferentes estudos científicos. 


\section{Critérios de elegibilidade}

A preferência foi para as pesquisas científicas sobre a ascensão da COVID-19, e para enriquecer a pesquisa investigou-se a transmissão da doença, as intercorrências e as demandas do sistema de saúde no Brasil e outros países. As pesquisas epidemiológicas, os estudos de relato de casos, os ensaios clínicos e os estudos clínicos de laboratório foram os de maior interesse dos pesquisadores. No entanto, outros tipos de estudos poderiam ser selecionados para possíveis comparativas com os nossos resultados obtidos, uma vez que apresentassem maiores correlações com o objetivo desta pesquisa. Subsequentemente, com o objetivo de melhorar a compreensão sobre as intercorrências da COVID-19 e as principais demandas do sistema de saúde, permitiu-se a inclusão de revisões sistemáticas para análise qualitativa.

Possibilitou-se ainda a complementação de informações atualizadas de jornais, cartas e cartilhas para completar as informações atualizadas no decorrer do texto. Entretanto, as mesmas não entraram na análise qualitativa, uma vez que o objetivo era apenas para enriquecer a pesquisa com dados atualizados. Da mesma forma, as pesquisas metodológicas com estratégias de técnicas para melhor elucidar os critérios de seleção da coleta de dados e esclarecimento de normas e estratégias para o desenvolvimento de revisões. Já outras escritas contidas em editoriais, opiniões pessoais, comentários e resumos de congressos não foram considerados para esta pesquisa.

Programou-se uma fonte de busca literária através de um diagrama de fluxo baseado no cheklist PRISMA ${ }^{9}$, nos idiomas em português e inglês. Utilizaram-se os descritores em Ciência da Saúde da Biblioteca Virtual em Saúde Lilacs (DeCS) para obtenção das palavras chave, nas bases de dados Web of Science, Scopus, PubMed/MEDLINE, Literatura Latino-Americana e do Caribe em Ciências da Saúde (LILACS), e biblioteca Cochrane. Foram selecionados os descritores, "coronavirus, pandemics, disease prevention" associados aos operadores booleanos "AND" e "OR", de modo a adquirir estudos mais aderentes ao tema pré-estabelecido.

\section{Seleção dos estudos e extração dos dados}

Os autores extraíram os dados mais relevantes a partir da obtenção dos estudos científicos nas respectivas bases de dados. Vale destacar que qualquer informação não bem esclarecida ou por falta de dados sobre as características dos estudos, os autores dos periódicos seriam contatados para melhores esclarecimentos.

Método de exclusão: um processo de seleção foi desenvolvido com as respectivas estratégias: leitura dos títulos; leitura dos resumos; estudos não relacionados principalmente ao Sars-CoV-2, que causa a doença COVID-19; estudos que não apresentaram as dificuldades do sistema de saúde; as demandas e divergências da prevenção da doença e percentuais de comprometimento. 
Critérios de inclusão: utilizou-se a estratégia $\mathrm{PICO}^{10}$. A população de interesse incluiu os pacientes assintomáticos e sintomáticos da COVID-19, com idade maior de 18 anos. A intervenção de interesse foram os métodos de isolamento e estratégias de assepsia e/ou higienização das diferentes populações. Entre os estudos, foram extraídos os dados e desfechos de interesse, contemplando as diferentes intercorrências e comprometimentos da COVID-19 e sua velocidade de progressão. Nos resultados, a verificação em números absolutos e relativos sobre as taxas de letalidade no cenário mundial, a resposta da vigilância nacional brasileira efetiva à doença do coronavírus, propagação do Sars-CoV-2 e as medidas de prevenção. Da mesma maneira, os parâmetros sobre as dificuldades de superar esse desafio no Brasil.

\section{Avaliação de viés nos diferentes estudos}

De modo geral tentava-se identificar se as informações eram suficientes, fatores de vieses de seleção, aferição, confundimento e poder do estudo. $O$ direcionamento dos autores, principalmente nos estudos de caso. Observou-se 0 período de incubação da COVID-19 e a similaridade entre os grupos em observação, uma vez que os desfechos deveriam ser condizentes com os objetivos propostos e as informações obtidas de maneira semelhante. Subsequentemente, a verificação sobre a proposta das diferentes pesquisas condizentes com os objetivos das mesmas. Observavam-se os diferentes direcionamentos, que permitissem uma maior informação sobre as propostas do protocolamento inicial da presente pesquisa. Portanto, conservaram-se as propriedades de investigação avaliando as particularidades, confiabilidade, validade dos dados e qualidade de evidência de forma similar em todos os estudos selecionados.

Após verificar novamente os critérios e adquirir os artigos a serem utilizados, reorganizamos o número de estudos selecionados em tópicos: comprometimento com as taxas de letalidade e transmissibilidade nos diferentes países; resposta da vigilância nacional efetiva à COVID-19 no Brasil; propagação dos patógenos humanos do SarsCoV-2 no cenário mundial e no Brasil; prevenção e recursos para interromper a transmissibilidade da COVID-19.

\section{RESULTADOS E DISCUSSÃO}

Na primeira etapa utilizaram-se os bancos de dados selecionados para procurar os artigos, 372 artigos foram identificados sobre o tema de interesse. Durante o processo de exclusão, 86 artigos foram eliminados por serem duplicados. Desta forma, 286 artigos em inglês e português foram selecionados. Concecutivamente, uma análise abrangente do título e do resumo eliminou 237. Totalizando 49 artigos elegíveis para a segunda etapa da revisão.

Na segunda etapa, 20 estudos científicos foram excluídos: oito (8) apresentavam uma população diferente do protocolo pré-estabelecido, cinco (5) estudos apresentaram poucos detalhes na seção de método e/ou pouca especificidade, cinco (5) pesquisas continham percentuais não bem elucidados sobre a propagação do coronavírus, 
e duas (2) publicações científicas não apresentaram nenhuma informação para avaliar os fatores relacionados às estratégias contra a COVID-19. Vinte nove (29) estudos foram incluídos na análise qualitativa, conforme Figura 1.

Figura 1. Fluxograma das atividades do processo de seleção através do diagrama de busca bibliográfica adaptado cheklist PRISMA.

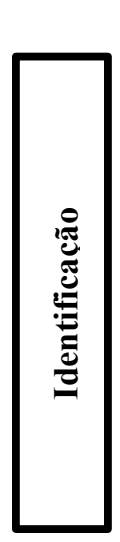

Identificação dos estudos nas bases de dados eletrônicos

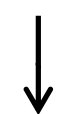

Busca em base de dados: total de referências identificadas $(n=372)$

Pubmed/MEDLINE (n=104) Web of Science ( $\mathrm{n}=101)$ Scopus (n=43) LILACS ( $\mathrm{n}=65)$ Cochrane $(\mathrm{n}=59)$

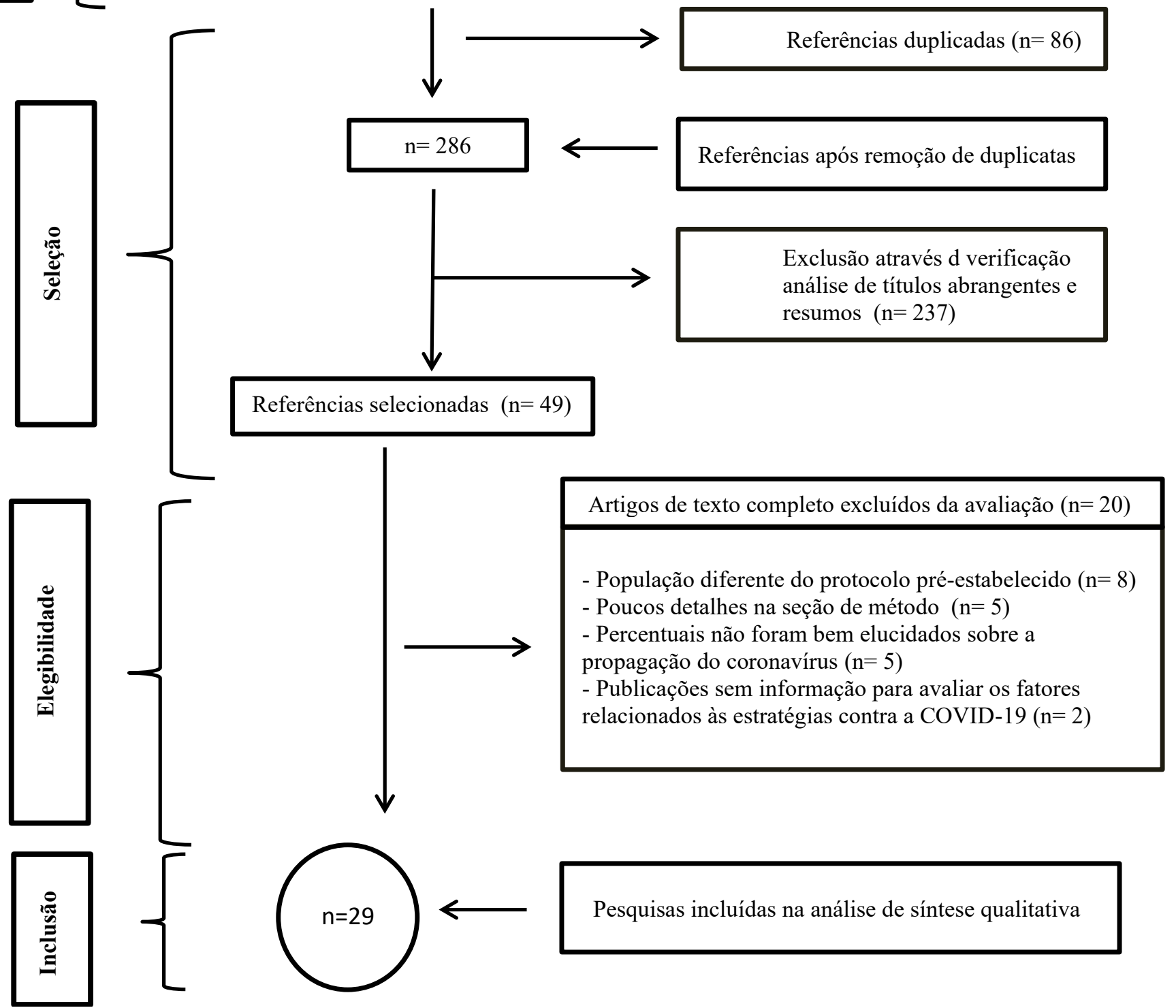


Na presente pesquisa, 29 estudos apresentaram maior correlação com os critérios de seleção e contemplaram os objetivos desta investigação, 16 (55,17\%) de estudos epidemiológicos, $6(20,68 \%)$ estudos de revisão, 4 (13,79\%) estudos de relato de caso, 2 (6,89\%) estudo clínico em laboratório, 1 (3,44\%) estudo de ensaio clínico, de acordo com a Figura 2.

Figura 2. Estudos selecionados nos critérios pré-estabelecidos.

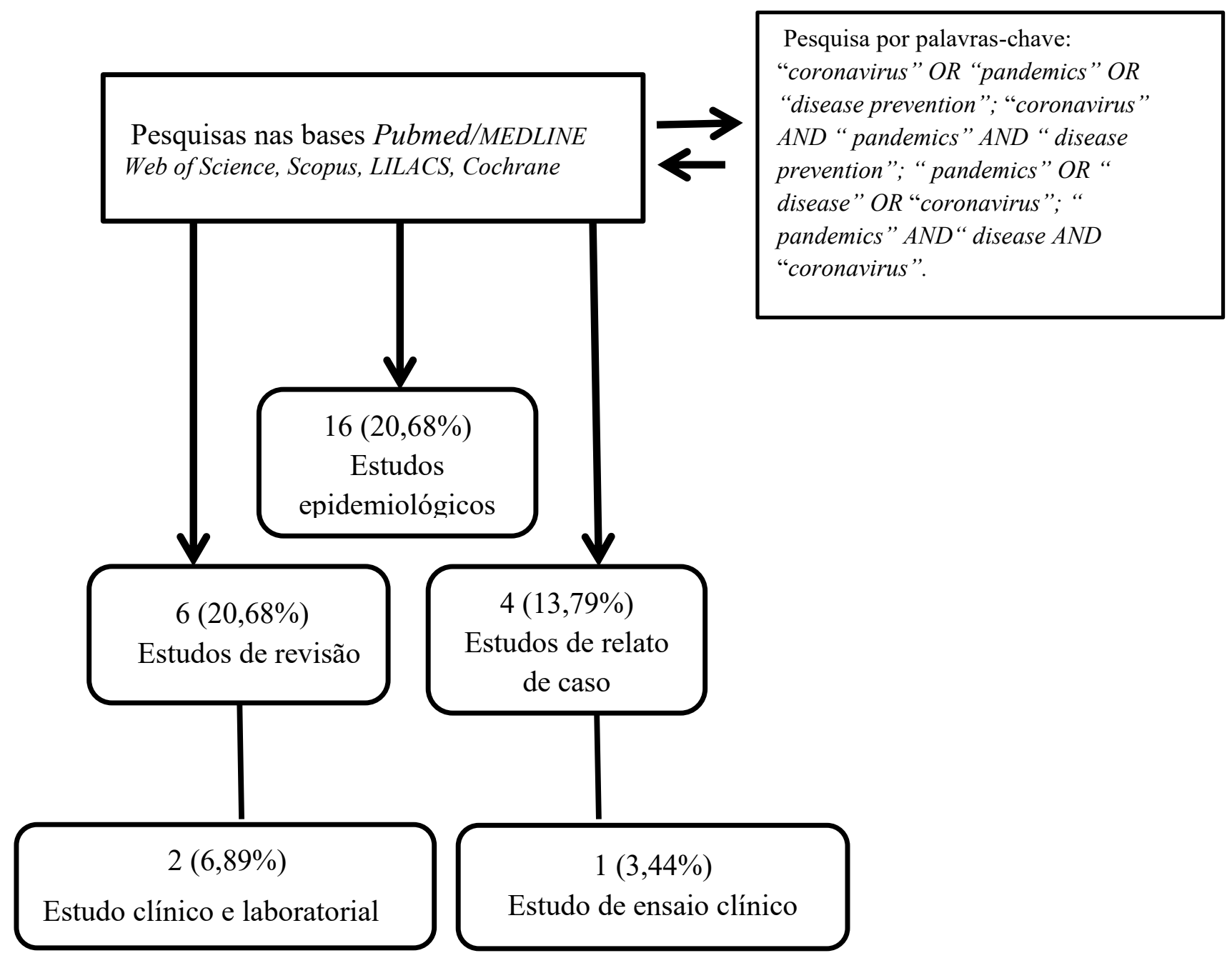

Desenvolveu-se uma comparação entre Sars-Cov e Sars-CoV-2. Os dados do Sars-Cov basearam-se nos números publicados de 1 de novembro de 2002 até os dados computados no dia 31 de julho de 2003, e os dados do Sars-CoV-2 desde 21 de janeiro de 2020 até os dados computados no dia 7 de abril de 2020, comparando os fatores clínicos, números absolutos e relativos de casos confirmados e letalidade, conforme a Tabela $1^{11,12}$. 
Tabela 1. Comparação tabular do Sars-Cov a partir de 1 de novembro de 2002 até os dados computados em 31 de julho de 200311 com relação ao Sars-CoV-2 a partir de 21 de janeiro de 2020 até os dados computados no dia 7 de abril de $2020^{12}$.

\begin{tabular}{|c|c|c|c|}
\hline Variáveis & $\begin{array}{c}\text { SARS-COV / dados } \\
\text { computados no dia } 31 \text { de } \\
\text { julho de } 2003\end{array}$ & $\begin{array}{c}\text { SARS-2CoV-2 / dados } \\
\text { computados no dia } 7 \text { de } \\
\text { abril de } 2020\end{array}$ & $\begin{array}{l}\text { Resultado das } \\
\text { diferenças }\end{array}$ \\
\hline $\begin{array}{l}\text { Período de } \\
\text { incubação do } \\
\text { vírus. }\end{array}$ & Dois a sete dias & Dois a quatorze dias & $\begin{array}{c}\text { SARS-2CoV-2 } \\
\text { apresenta um período } \\
\text { de incubação maior }\end{array}$ \\
\hline $\begin{array}{l}\text { Aspectos } \\
\text { clínicos }\end{array}$ & Febre, tosse seca, falta de ar & Febre, tosse, falta de ar & $\begin{array}{l}\text { Tosse seca no SARS- } \\
\text { COV demarca uma } \\
\text { diferença entre as duas }\end{array}$ \\
\hline $\begin{array}{l}\text { Total de } \\
\text { casos } \\
\text { confirmados }\end{array}$ & 8.096 & 1.348 .488 & $\begin{array}{l}\text { Propagação mais } \\
\text { rápida no SARS- } \\
\quad 2 \mathrm{CoV}-2\end{array}$ \\
\hline $\begin{array}{l}\text { Número de } \\
\text { mortos. } \\
\text { Dados } \\
\text { globais. }\end{array}$ & 774 & 77.643 & $\begin{array}{l}\text { Maior letalidade no } \\
\text { SARS-2CoV-2 }\end{array}$ \\
\hline $\begin{array}{l}\text { Total de } \\
\text { mortalidade } \\
\text { em números } \\
\text { relativos. }\end{array}$ & $9,56 \%$ & $5,75 \%$ & $\begin{array}{l}\text { Números relativos } \\
\text { com ascensão vertical } \\
\text { mais rápida no SARS- } \\
\quad 2 \mathrm{CoV}-2\end{array}$ \\
\hline $\begin{array}{l}\text { Total de } \\
\text { casos } \\
\text { confirmados } \\
\text { no Brasil }\end{array}$ & $\mathrm{DNC}$ & 11.982 & - \\
\hline $\begin{array}{l}\text { Número de } \\
\text { mortos. } \\
\text { Dados no } \\
\text { Brasil. }\end{array}$ & $\mathrm{DNC}$ & 540 mortos & - \\
\hline $\begin{array}{l}\text { Mortalidade } \\
\text { em números } \\
\text { relativos no } \\
\text { Brasil. }\end{array}$ & DNC & $4,5 \%$ & - \\
\hline
\end{tabular}

DNC- Dados não computados 


\section{Taxas de letalidade e parâmetros de transmissibilidade}

De acordo com a Organização Mundial de Saúde (OMS) a COVID-19 deixou 10-15\% dos pacientes em uma situação grave e em 2 de março de 2020 já havia 2.915 mortes $^{13}$. A taxa de letalidade da COVID-19 é de 3,4\%, sendo que este percentual é mais elevado na China, em comparação com os outros países ${ }^{14}$. Embora $80 \%$ dos casos são leves, a COVID-19 em 2020 supera a letatidade da Influenza A H1N1, em um percentual previsto para COVID-19 ente 0,5 a 4\% versus 0,2\% da H1N1 em 2009 ${ }^{15}$. Da mesma forma, a pandemia da Influenza sazonal em 1918 (2\%) e 1957 $(0,6 \%)^{16}$. O prognóstico poderá superar a gripe espanhola em 1919 , que atingiu $3 \%$ da letalidade no mundo ${ }^{17}$.

Dentre os patógenos humanos do coronavírus, a epidemia da síndrome respiratória aguda grave (SRAG), que surgiu em 2003 em Hong Kong (China), com uma letalidade de 10\%, e a síndrome respiratória do Oriente Médio, que emergiu na Arábia Saudita em 2012 e apontou 30\% da letalidade ${ }^{18,19}$. Em uma busca aleatória de diferentes países, na China, em 2 de março de 2020 havia 80.174 de casos confirmados com 2.915 mortos pela COVID-19. Em 7 de abril eram 83.137 casos confirmados com 3.340 , apresentando um percentual de $3 \%$ para $4 \%$, com um aumento de $1 \%$ de letalidade. Já na Itália, no mesmo período, o aumento foi de 1,82\% para 12,6\%, em 7 de abril de 2020, apontanto uma ascensão de 10,78\%, confirmando em números absolutos 17.161 mortos. Na República da Coréia, de 5,67\% reduziu para 1,85\% e, na Alemanha, de $0 \%$ para $1,72 \%$ conferindo 1.780 óbitos. No contimente americano, a maior alta foi nos Estados Unidos da América (EUA), demonstrando um aumento entre 2 de março e 7 de abril de 0\% para 10,76\%, anunciando 10.760 óbitos. No México, de 0\% aumentou para 4,54\% com 109 mortos confirmados e, no Brasil, de 0\% elevou-se para $4,5 \%$, indicando números absolutos de 540 mortos $^{12,13}$.

Apoiando-se em achados científicos, o fator relacionado à dinâmica na detecção de casos da COVID-19 confirma ser uma variável fundamental na desaceleração de sua contaminação. Uma afirmativa consistente, sendo que a Alemanha reconheceu o seu surto com antecedência. Logo, concluiu-se que testes mais rápidos a serem desenvolvidos com as respectivas confirmações são imprescindíveis e, caso contrário, pode favorecer a sua propagação. Como se observou na Coréia do Sul, no período de 1 a 20 de março de 2020, uma ascendência de 0,6\% da letalidade pela COVID-19 (em um curto período de tempo). Todavia, em 7 de abril a Coréia reduziu o seu percentual de óbitos em 3,82\% 12,13 .

Contudo, os achados dificultam as comparativas diretas entre os países. Dentre outras, na Coréia, a média de idade da população acima de 60 anos é de $14 \%$, e na Itália $26 \%$ da população apresenta mais de 65 anos. Em contrapartida, a Alemanha apresentou uma diferença excepcionalmente baixa de mortos em comparação com a Itália no período de 2 de março a 7 de abril, surpreendendo e dificultando os resultados advindos da Itália, que favoreceram a concepção sobre a alta faixa etária associada ao aumento da letalidade pela COVID-19, uma vez que 25\% da popução alemã tem mais de 65 anos de idade $\mathrm{i}^{1,2,6,12,13}$. 


\section{Resposta da vigilância nacional efetiva à COVID-19 no Brasil}

De acordo com a Organização mundial de Saúde (OMS) o suporte de Logística de Operações (OSL) vem convocando simultâneas reuniões às principais partes interessadas, incluindo fabricantes de ventiladores respiratórios, acessórios e equipamentos para países de maior vulnerabilidade. As demandas são maiores que a procura e os índices são crescentes de letalidade e os percentuais aumentam de forma assustadora².

No Brasil o número de infectados é crescente a cada dia e ao Laboratório Central de Saúde Pública (LACEN) caberia, no seu âmbito da vigilância laboratorial, conter materiais fundamentais para a manipulação de amostras recebidas e, subsequentemente, a liberação de resultados no tempo apropriado. No entanto, o fator atraso na liberação dos resultados parece apresentar uma forte relação com a falta de equipamentos e a ausência de profissionais mais capacitados $^{20}$. Em 31 de janeiro de 2020, a vigilância do Ministério da Saúde do Brasil implantou o Grupo de Trabalho Interministerial de Emergência em Saúde Pública de Importância Nacional e Internacional, com o propósito de conduzir a situação e estabelecer protocolos de ação para a vigilância do Sars-Cov-2 no país²1 .

Entretanto, vale ressaltar que além da falta de equipamentos mais três fatores parecem dificultar a entrada de resultados no tempo oportuno: o primeiro é que antes do aparecimento do Sars-Cov-2 no Brasil, o protocolo de vigilância de SRAG não incluía o COVID-19 como rotina laboratorial de exames de vigilância, cujo mesmo apenas era explorado em situações de óbitos e custos através dos Laboratórios Nacionais de Influenza (NICs). O segundo fator é a validação dos dados ao serem digitados, uma vez que em algumas localidades este preenchimento, até este momento, se caracteriza pelo uso de fichas em papel que necessitam de digitação. 0 terceiro problema são as dificuldades de integração entre as fontes de informações dentro do próprio país.

No Brasil, por exemplo, existe a necessidade de uma melhora na intercomunicação entre o Sistema de Informação de Agravos de Notificação (SINAN) e o Sistema Gerenciador de Ambiente Laboratorial (GAL), que por sua vez, deveria alcançar números mais precisos de notificações realizadas pelo SINAN, proporcionando os dados com maior exatidão e melhorando a dinâmica de percepção sobre a velocidade de propagação de uma nova doença e aderindo os princípios de um sistema epidemiológico de maior precisão $0^{20}$.

\section{Propagação dos patógenos humanos do Sars-Cov-2 no cenário mundial e no Brasil}

Na primeira semana de fevereiro do ano de 2020 diferentes países da América já haviam importado a COVID-19, incluindo Canadá e Estados Unidos. No Brasil, já havia 9 casos da COVID-19 sendo investigados ${ }^{22}$. 0 número básico de reprodução $(R 0)$ é uma forma de avaliar a velocidade de propagação de uma doença, que identifica-se através do número médio de casos secundários por caso primário ${ }^{5,22}$. Em uma comparativa com a transmissão da COVID-19 e 0 vírus da Influenza A H1N1, ocorrido no ano de 2009, o R0 apontou parâmetros superiores para a COVID-19, de 1,6 a 4,1 versus 1,3 a 1,8 , respectivamente ${ }^{23,24}$. 
A avaliação de casos em Wuhan, na China, no período de 1 a 22 de janeiro de 2020, apresentou um percentual de 5,0\% (3,6-7,4); 21022 (11090-33490) total de infecções ${ }^{5}$. Os preditores para internações e mortalidade da Sars-Cov-2 vem apresentando uma transmissibilidade maior e sugere-se uma taxa de infectados que será superior a Influenza A H1N1. A precisão destes dados e/ou afirmativas depende da proporção de casos graves e o número de mortos, uma vez que ainda são desconhecidos. Já as taxas de SARG pela Influenza A H1N1 alcançou um percentual de óbitos de $20 \%$ no Brasil| ${ }^{24}$.

Na Sars-Cov-2 o comprometimento maior está relacionado aos pacientes com idade acima de 60 anos e possíveis comorbidades que atingem o sistema imunológico do idoso ${ }^{25}$. 0 poder dos testes, 0 apoio financeiro e as informações científicas são fatores indispensáveis para um prognóstico promissor à cura da Sars-Cov-2. Na China, Read et al. ${ }^{5}$ utilizaram um modelo de transmissão em Wuhan, confirmando que um número reprodutivo básico de OR=3,11 (IC 95\%, 2,39-4,13); 58-76\% das transmissões devem ser impedidas para evitar o aumento.

Embora no Brasil houve um certo avanço na vigilância epidemiológica para vírus precedentes e desenvolvimento de vacinas ${ }^{26}$, o nível cultural de um população parece ser um fator fundamental neste novo obstáculo a ser vencido. No Brasil, o potencial de chegada do novo vírus se defrontou com um momento em que existe uma redução de recursos para o Sistema Único de Saúde (SUS), fragilizando as pesquisas científicas para as possíveis respostas e detecção antecipada da doença ${ }^{20}$. Ainda que exista o privilégio sobre uma dinâmica rápida de publicações científicas sobre o tema coronavírus nos principais sites de pesquisa e um avanço no uso de mídias como meios de divulgação, também existem variáveis que dificultam o processo de reeducação da população brasileira, como os áudios falsos com recomendações equivocadas na mídia social, que prejudicam o redirecionamento correto dos profissionais especializados em saúde ${ }^{20,27}$.

Mesmo que nos países da América Latina exista uma concepção dos gestores em tentar intensificar a preparação dos profissionais para enfrentar o novo coronavírus, será necessário intensificar o treinamento e comunicação intracontinental e intercontinental ${ }^{28}$. Dentre outros fatores que dificultam estas relações estão a heterogeneidade política e as dificuldades econômicas, pois existem regiões com baixo índice humano e dificuldades nas relações sociais e políticas ${ }^{28,29,30}$.

\section{Prevenção e recursos para interromper a transmissibilidade da COVID-19}

Para reduzir as taxas de transmissibilidade do novo vírus, revela-se a necessidade de uma reeducação da população brasileira em um processo de medidas efetivas que requer as seguintes condutas: proteger o profissional de saúde com os adequados equipamentos de proteção individual, identificação dos pacientes sintomáticos, isolando-os após os devidos testes e identificar os envolvidos para uma subsequente quarentena ${ }^{31}$. Condizente com a China, que parece ter encontrado a forma de impedir a proliferação da letalidade e torna-se um exemplo para os demais países, já 
que os dois principais procedimentos foram a proteção dos profissionais da saúde e o ato de se desenvolver uma conduta de isolamento da população, impedindo a disseminação da doença.

A implantação de melhores estruturas para permitir o isolamento dos pacientes sintomáticos é um recurso que necessita uma nova concepção e/ou novas diretrizes dos gestores governamentais, que na atual realidade pandêmica lutavam por um maior crescimento econômico. Da mesma maneira, a necessidade de aumentar o número de ventiladores respiratórios para os casos de novas internações que envolvem análises e parâmetros estatísticos, baseados em dados advindos de outros países, correlacionando percentuais necessários de equipamentos, conforme as necessidades do Brasil|31,32,33.

Sobre os determinantes sociais, embora as estratégias adotadas de isolamento social, fechamento de escolas e universidades sejam decisões fundamentais e importantes para o controle da mobilidade populacional, o processo gerou outros impactos que resultaram em sentimentos de tédio, desamparo e abandono na vida das pessoas ${ }^{34,35,36}$. 0 aspecto mais atingido foi o convívio social. Em uma pesquisa com 16.440 indivíduos, 45\% das pessoas com alta renda e de maior escolaridade demarcaram a falta do convívio social ser um grande problema. Já nos de baixa renda e menor escolaridade, os maiores impactos direcionaram-se aos problemas financeiros, compreendendo $35 \%$ dos entrevistados. Sobre o estresse, os praticantes de esportes são os menos afetados, 13\% e, 50,3\% dos que praticam atividade física, revelaram ter um sono normal durante a pandemia. Vale ressaltar que $73 \%$ dos indivíduos com poucas condições de moradia responderam terem menos disposição de permanecerem isolados ${ }^{36}$.

Com os estudos científicos estritamente limitados e/ou ensaios clínicos ainda não finalizados, os desfechos de uma promissora vacina para mudar o crescimento da transmissibilidade tornou-se uma busca incansável no cenário mundial. Desta forma, o que prevalece no Brasil é uma carência de estratégias diante da atual situação, dentre outros fatores, um grande número de profissionais destreinados, e as sugestões metodológicas são dificuldades a serem conquistadas. No governo, o sentimento de fazer parte de um processo de cuidados coletivos e repensar o impacto de seus atos, considerando como prioridade as ações sociais e a desaceleração da doença ${ }^{36}$. Nos hospitais, o treinamento adequado das equipes e, para as crianças, a reeducação e desenvolvimento de atividades de lazer para se estabelecer a rotina tranquila dentro das casas $^{34}$. Outro aspecto que parece somar na interrupção da pandemia é reduzir o pânico da população brasileira, pois assim como o Sars-Cov-2, a ciência vem progredindo velozmente. Logo, a reversão deste quadro dependerá exclusivamente da confiança em nossos profissionais da saúde e na força de reeducação das pessoas, intensificando a mobilização social, que terá no futuro a herança de uma maior conscientização. 


\section{CONSIDERAÇÕES FINAIS}

A COVID-19 apresenta uma rápida ascensão e a missão que se atribui aos nossos governantes parece ser a reavaliação para converter a ascensão vertical. Profissionais capacitados a exposição de agentes patogênicos, estratégias de aconselhamento psicológico e equipes multidisciplinares com maior preparo dificultam a desaceleração do coronavírus no Brasil. É necessário preconizar o destino das verbas para a capacitação de profissionais, e novos equipamentos que permitam o acompanhamento dos resultados no período do dia e/ou no tempo real, uma vez que no atual cenário prevalece uma imprecisão de sua interrupção e o não decréscimo nas taxas de letalidade. Portanto, é de fundamental importância a ação de resguardo à segurança dos profissionais e agilizar a identificação dos pacientes sintomáticos à COVID-19 e dos indivíduos envolvidos, posto que a sua disseminação e/ou celeridade epidemiológica deve ser contida com a mesma força que ela é produzida.

\section{REFERÊNCIAS}

1. Zhu N, Zhang D, Wang W, et al. A novel coronavirus from patients with pneumonia in China, 2019. N Engl J Med 2020; 382: 727-33.

2. Johns Hopkins University. Coronavirus COVID-19 global cases by Johns Hopkins CSSE. https://gisanddata. maps.arcgis.com/apps/opsdashboard/index.html\#/bda7594740fd40299423467b48e9ecf6 (acessado em 04/março /2020).

3. Fink S. Worst-case estimates for U.S. coronavirus deaths. New York Times. March 18, 2020. https:// www.nytimes.com/2020/03/13/us/coronavirus-deaths-estimate.html

4. Jacobs A, Richtel M, Baker M. 'At war with no ammo': doctors say shortage of protective gear is dire. New York Times. March 19, 2020. https://www.nytimes.com/2020/03/19/healt

5. Read JM, Bridgen JRE, Cummings DAT, Ho A, Jewell CP. Novel coronavirus 2019-nCoV: early estimation of epidemiological parameters and epidemic predictions. medRxiv 2020; 28 jan. https://www.medrxiv.org/content/10. $1101 / 2020.01 .23 .20018549 v 2$ 
6. Rothe C, Schunk M, Sothmann P, et al. Transmission of 2019-NCOV infection from an asymptomatic contact in Germany. N Engl J Med. 2020;382(10):970-971. doi:10.1056/NEJMc2001468.

7. Callaway E, Cyranoski D. China coronavirus: six questions scientists are asking. Nature 2020; 577: 605-7.

8. Cavenett. detection of Wuhan coronavirus 2019 by real-time RT-PCR. Geneva: World Health Organization, January 13, 2020. 2020;5. doi:10.1017/CBO9781107415324.004.

9. Galvão TF, Pansani TSA HD. Principais itens para relatar Revisões sistemáticas e Meta-análises: A recomendação PRISMA. Epidemiol. 2015;24(2):335-42. http://www.scielo.br/scielo.php?script=sci_arttext\&pi$d=S 223796222015000200335$.

10. Santos CMCS, Pimenta CAM NM. A estratégia PICO para a construção da pergunta de pesquisa e busca de evidências. Latino-Am. 2007;15(3):508-511. http://www.scielo.br/scielo.php?pid=s0104=11692007000300023-\&scriptsci_abstract\&tlng=pt.

11. Organização Mundial da Saúde (2003) Resumo de prováveis casos de SARS com início da doença de 1 de Novembro 2002 a 31 de Julho de 2003. https://www.who.int/csr/sars/country/table2003_09_23/en/.

12. World Health Organization .Coronavirus disease 2019. i. 2020;2019(April):2633. https://www.who.int/ emergencies/diseases/novel-coronavirus-2019/situation-reports

13. World Health Organization. Coronavirus disease 2019 (COVID-19) Situation Report - 42. Available at: https://www.who.int/emergencies/diseases/novel-coronavirus-2019/situation-reports/. (acessado em 29 mar. 2020)

14. World Health Organization. WHO Director General's opening remarks at the media briefing on COVID-19 - 3 March 2020 [Internet]. World Health Organization; 2020. https:// www.who.int/dg/speeches/detail/who directorgeneral-s-opening-remarks-at-the-media-briefing-oncovid-19---20-march-2020 (acessado em 29 mar. 2020) 
15. Khandaker G, Dierig A, Rashid H, King C, Heron L, Booy R. Systematic review of clinical and epidemiological features of the pandemic influenza A (H1N1) 2009. Influenza Other Respir Viruses 2011; 5(3): 148-56.

16. Zhonghua Liu Xing Bing Xue Za Zhi. The epidemiological characteristics of an outbreak of 2019 novel coronavirus diseases (COVID-19) in China. 2020 Feb 17;41(2):145-151.

17. World Health Organization. Severe acute respiratory syndrome (SARS). https://www.who.int/csr/sars/en/ (acessado em 08/Fev/2020).

18. World Health Organization. Pandemic Influenza preparedness and response: a WHO guidance document [Internet]. Geneva: World Health Organization; 2009 (acessado em 8 de abril 2020).

19. World Health Organization. Prioritizing diseases for research and development in emergency contexts. https://www.who.int/activities/prioritizing-diseases-for-research-and-development-in-emergency-contexts (acessado em 29/ Jan/2020). » https://www.who.int/activities/prioritizing-diseases-for-research-and-development-in-emergency-contexts.

20. Lana RM, Coelho FC, Gomes MF da C, et al. Emergência do novo coronavírus (SARS-CoV-2) e o papel de uma vigilância nacional em saúde oportuna e efetiva. Cad Saude Publica. 2020;36(3):e00019620. doi:10.1590/ 0102-311x00019620.

21. Ministério da Saúde. Coronavírus e novo coronavírus: o que é, causas, sintomas, tratamento e prevenção. https://www.saude.gov.br/ saude-de-a-z/coronavirus (acessado em 10/ março/2020).

22. Secretaria de Vigilância em Saúde, Ministério da Saúde. Infecção humana pelo novo coronavírus (2019nCoV). Boletim Epidemiológico 2020; (02). https://portalarquivos2.saude.gov.br/images/pdf/2020/fevereiro/07/BE-COE-Coronavirus-n020702.pdf » https://portalarquivos2.saude.gov.br/images/pdf/2020/fevereiro/07/BE-COE-Coronavirus-n020702.pdf.

23. Liu T, Hu J, Kang M, Lin L, Zhong H, Xiao J, et al. Transmission dynamics of 2019 novel coronavirus (2019-nCoV). BioRxiv 2020; 26 jan. https://www.biorxiv.org/content/10.1101/2020.01.25.919787v1. 
24. InfoGripe. Situação da gripe. http://info.gripe.fiocruz.br/ 2020. (acessado em 03 fev 2020)

25. World Health Organization. Q\&A on coronaviruses. https://www.who.int/news-room/q-a-detail/q-a-coronaviruses (acessado em 10/Fev/2020). https://www.who.int/news-room/q-a-detail/q-a-coronaviruses

26. Costa LMC, Merchan-Hamann E. Influenza pandemics and the structure of Brazilian health care system: brief history and characterization of the scenarios. Rev Pan-Amazônica Saúde 2016; 7:1125. doi: 10.5123/S2176-62232016000100002. http://scielo.iec.gov.br/scielo.php?script=sci_abstract\&pi$d=\$ 2176-62232016000100002 \&$ Ing=en\&nrm=isso

27. Johns Hopkins University. Coronavirus COVID-19 global cases by Johns Hopkins CSSE. https://gisanddata.maps.arcgis.com/apps/opsdashboard/index.html\#/bda7594740fd40299423467b48e9ecf6 (acessado em 04 fev 2020).

28. Mondolfi Paniz-AE, Tami A, Grillet ME, Marquez M, Hernandez-Villena J, Rodriguez MA-Escalona et all. Ressurgimento de doenças evitáveis em- Vaccine Venezuela como uma ameaça à saúde pública regional nas Américas. Emerg Infect Dis. 2019; 25: 2019:625-32.

29. Page KR, Doocy S, Reyna Ganteaume F, Castro JS, Spiegel P. crises de saúde pública do Beyrer C. Venezuela: uma emergência regional. Lancet. 2019; 393: 1254-1260.

30. Grillet ME, Hernandez-Villena JV, Llewellyn MS, Paniz-Mondolfi AE, Tami A, Vincenti-Gonzalez MF et all. Venezuela é crise humanitária, ressurgimento de doenças transmitidas por vetores, e Implicações para transbordamento na região. Lancet. 2019:2019.

31. Moura A. Sobre a possibilidade de interrupção da epidemia pelo coronavírus ( COVID-19) com base nas melhores evidências científicas disponíveis. 2020:1-3. doi:10.1590/1980-549720200021.

32. Fauci AS, Lane HC, Redfield RR. Covid-19 - Navigating the Uncharted. N Engl J Med. 2020. https://doi. org/10.1056/NEJMe2002387. 
33. Guan W-J, Ni Z-Y, Hu Y, Liang W, Ou C-Q, He J-X, et al. Clinical Characteristics of Coronavirus Disease 2019 in China. N Engl J Med. https://doi.org/10.1056/ NEJMoa2002032

34. Ornell F, Schuch JB, Sordi AO, Kessler FHP. Pandemia de medo e COVID-19: impacto na saúde mental e possíveis estratégicas. Revista Debates in Psychiatry. Disponível em: http://www.ufrgs.br/ufrgs/noticias/arquivos/pandemia- de-medo-e-covid-19-impacto-na-saude-mental-e-possiveis-estrategias.

35. Shigemura J, Ursano RJ, Morganstein JC, Kurosawa M, Benedek DM. Public responses to the novel 2019 coronavirus (2019-nCoV) in Japan: mental health consequences and target populations. Psychiatry Clin Neurosci. 74: 277-283, 2020. https://onlinelibrary.wiley.com/doi/epdf/10.1111/pcn.12988

36. Bezerra ACV, da Silva CEM, Soares FRG, da Silva JAM. Factors associated with people's behavior in social isolation during the covid-19 pandemic. Cienc e Saude Coletiva. 2020;25:2411-2421. doi:10.1590/141381232020256.1.10792020. 